\title{
Apparent Diffusion Coefficient
}

National Cancer Institute

\section{Source}

National Cancer Institute. Apparent Diffusion Coefficient. NCI Thesaurus. Code C116753.

A measurement of the magnitude of diffusion of water molecules within tissue. 